\title{
VALIDACIÓN DE UN CUESTIONARIO PARA LA EVALUACIÓN DEL APRENDIZAJE EN EL ALUMNO UNIVERSITARIO
}

\author{
Josefa Rodríguez Pulido \\ Josué Artiles Rodríguez \\ $\mathbf{M}^{\mathbf{a}}$ Victoria Aguiar Perera \\ Universidad de Las Palmas de Gran Canaria
}

RESUMEN: Este artículo aborda el aprendizaje del alumnado universitario en base a la utilización de estrategias y/ o enfoques, a la distribución de los tiempos de estudio, recursos, etc.; es decir, nos interesa saber de qué forma afrontan y gestionan el estudio los estudiantes para obtener el rendimiento académico que esperan alcanzar. El objetivo del presente estudio ha sido diseñar y analizar la validez de un cuestionario que reúna los requisitos metodológicos y psicométricos necesarios para evaluar el afrontamiento del aprendizaje en estudiantes universitarios. La validación del instrumento se ha llevado a cabo mediante juicio de expertos y análisis factorial confirmatorio del modelo teórico previo, siendo la estructura factorial de esta herramienta fiable y con un buen ajuste. Los análisis realizados han confirmado y mostrado cinco dimensiones o factores en el aprendizaje del alumno universitario: estrategia metodológica, progreso de los resultados, entornos educativos y de aprendizaje, organización de los tiempos, atribuciones en los resultados. El resultado final fue un cuestionario formado por 30 ítems en el que los mismos se valoran en una escala tipo Likert, de cinco opciones de respuesta, siendo $1=$ "Muy en desacuerdo" y 5= "Muy de acuerdo".

PALABRAS CLAVE: estudiantes universitarios, estudio y validación psicométrica, instrumento de evaluación, modelos de aprendizaje.

\section{VALIDATION OF A QUESTIONNAIRE FOR THE EVALUATION OF LEARNING IN THE UNIVERSITY STUDENT}

ABSTRACT: This article addresses the learning of university students based on the use of strategies and / or approaches, the distribution of study times, resources, etc.; that is, we want to know how the students approach and manage the study to obtain the academic performance they hope to achieve. The objective of this study was to design and analyze the validity of a questionnaire 
that meets the methodological requirements and psychometric requirements to assess the coping of learning in university students. The validation of the instrument has been carried out through expert judgment and confirmatory factor analysis of the theoretical model, with the factorial structure of this tool being reliable and with a good fit. The analyzes carried out have confirmed and demonstrated the dimensions or problems in the learning of the university student: methodological strategy, progress of the results, educational and learning environments, organization of the times, attributions in the results. The final result was a questionnaire made up of 30 items in which they are assessed on a Likert-type scale, with five response options, being $1=$ "Strongly disagree" and $5=$ "Strongly agree".

KEYWORDS: Learning models, study and psychometric validation, evaluation instrument, University.

Recibido: 04/02/2020

Aceptado: 18/12/2020

Correspondencia: Josefa Rodríguez Pulido, Facultad de Formación del Profesorado, Universidad de Las Palmas de Gran Canaria, Juan de Quesada, 30, 35001 Las Palmas de Gran Canaria. Email: josefa.rodriguez@ulpgc.es

\section{INTRODUCCIÓN}

El alumnado universitario orienta su aprendizaje hacia los resultados o hacia el propio proceso de aprendizaje (Cardozo, 2008). El aprendizaje orientado hacia el resultado hace referencia a la meta final del estudiante que persigue una buena nota, o no ser suspendido, diferente a la meta orientada al aprendizaje por sí mismo y a su disfrute (García, 2005; Calatayud, 2017). "Son varias las causas que diferencian a los buenos estudiantes de los que no lo son tanto: inteligencia, personalidad, conocimientos previos, motivación, cantidad y calidad de las estrategias que los alumnos ponen en juego cuando aprenden, etc." (García-Valcárcel y Tejedor, 2017).

Al hablar de aprendizajes entendemos que es un proceso que requiere de una acción determinada por parte del alumnado, para el logro de conocimientos, habilidades y valores. Ello hace que los estilos de aprendizaje se entiendan como variables personales del alumnado que explican las diferentes formas de abordar, planificar y responder de ellos ante las demandas del aprendizaje (Camarero, Martín del Buey y Herrero, 2000). También juegan un papel importante las estrategias didácticas que ponga en juego el profesorado a la hora de orientar su aprendizaje, al ser éstas los procedimientos utilizados por el alumnado con una intencionalidad determinada, ya que una acción educativa siempre está encaminada hacia una intencionalidad determinada (Coll y Bolea, 1990; Vivas, 2010).

Cuando el alumnado se enfrenta a la tarea como una amenaza, generando tensiones y actitudes negativas hacia el aprendizaje se encuentra condicionado por el resultado (García, 2005). Es decir, las calificaciones y/o los errores cometidos son 
vistos como un peligro, en lugar de como una oportunidad. Es necesario prestar importancia a la motivación intrínseca que puede generar el aprendizaje en sí mismo, pero, de la misma forma, también es necesario partir y tener en cuenta de la motivación que pudiera generar el profesorado (Elizondo, Rodríguez y Rodríguez, 2018).

El Espacio Europeo de Educación Superior proporciona un entorno donde se desarrollan metodologías activas, orientando la docencia hacia el aprendizaje y no solo a la calificación, por lo tanto, esto debería materializarse en un cambio en la orientación de la evaluación de manera que no se enfoque tanto en los resultados y sí en la tarea de aprender (Padilla y Gil, 2008). Aun así, la evaluación esperada, entendida por el resultado final, es la que más puede condicionar al estudiante, dado que es un requisito para solventar la materia (Morales, 2006).

Todas las personas tienen un perfil de estilo de pensamiento y un estilo de aprendizaje propio que es desarrollado a través del uso de diversas estrategias (Mosquera, 2012). Dichas estrategias de aprendizaje son entendidas como el conjunto organizado de lo que hace el alumnado para alcanzar un aprendizaje en un contexto social determinado, es decir, operaciones o tareas de razonamiento y resolución de problemas (Gargallo, Almerich, Suárez-Rodríguez y García-Félix, 2012; Camarero Suárez, Buey y Herrero Diez, 2000). El hecho de aproximarnos a la identificación de las mismas, nos ayudará al entendimiento de los procesos de aprendizajes (Gargallo, Sánchez Peris, Ros y Ferreras, 2010).

Dicho lo anterior, son pocos los instrumentos de medida que se emplean para evaluar las estrategias de aprendizaje de los alumnos universitarios y, los que existen, presentan diversas limitaciones (Díaz y Fernández, 2002; Gargallo, Suárez-Rodríguez y Pérez-Pérez, 2009; Gargallo et al., 2015; Monereo et al., 2000; Rodríguez y Remesal, 2007); por ello, nos propusimos crear un instrumento que evaluara, de forma válida y fiable, los modos o las formas que tiene el alumnado universitario de afrontar el aprendizaje, partiendo de un modelo teórico del aprendizaje.

Por tanto, se pretende estudiar la validez del cuestionario diseñado a través de juicio de expertos, de análisis de fiabilidad y confirmar la multidimensionalidad de constructo del modelo teórico previo mediante Análisis Factorial Confirmatorio (AFC). Este último estadígrafo, que es una aplicación de los modelos de ecuaciones estructurales, es muy empleado en las ciencias sociales para validar las estructuras factoriales de las variables a estudiar, identificar las dimensiones que subyacen sobre los factores originales y reducir así el número de los mismos con la pérdida mínima de información, comprobar las relaciones de dependencia de las variables observadas, calcular el ajuste de bondad del modelo, etc. (Carrión, 1984; Escobar y Cuervo, 2008; Freiberg et al., 2013; Jöreskog, 1969; Lloret-Segura et al., 2014; Pérez, Chacón y Moreno, 2000).

Asimismo, los datos que se desprendan se someterán a AFC atendiendo a las principales medidas de comprobación: pesos o saturaciones factoriales, coeficiente de fiabilidad e índices de ajuste de bondad del modelo (Cronbach, 1990; García-Cueto y Miranda, 1998; Varela Mallou, Abalo Piñeiro, Rial Boubeta y Braña Tobio, 2006; Moral, 2006). Pero, además, se atenderá a la prueba de adecuación muestral para comprobar si la relación entre las variables es satisfactoria, con lo cual el modelo factorial sería significativo, y la de esfericidad, para conocer la aplicabilidad del análisis 
factorial de las variables estudiadas. Para el cálculo de ajuste del modelo se utilizará la medida de extracción máxima verosimilitud y el método de rotación varimax con normalización Kaiser (Kaiser, 1977; Pérez, 2005; Pindick y Rubinfeld, 2001; Uriel y Manzano, 2002; Ximénez y García, 2005).

Por todo lo indicado nos planteamos diseñar y analizar la validez de un cuestionario que reúna los requisitos metodológicos y psicométricos necesarios para evaluar el afrontamiento del aprendizaje en el estudiante universitario. Los análisis realizados han confirmado y mostrado cinco dimensiones o factores en el aprendizaje del alumno universitario en la Escala de Valoración del Aprendizaje del Alumnado Universitario (EVAAU): estrategia metodológica, progreso de los resultados, entornos educativos y de aprendizaje, organización de los tiempos, atribuciones en los resultados.

\section{Método}

\subsection{Objetivos}

El objetivo de este estudio, que forma parte de una investigación más amplia y de mayor alcance, se centra en comprobar la estructura factorial y la fiabilidad de un cuestionario cuyo uso atiende la evaluación de las estrategias que utilizan los estudiantes universitarios en el aprendizaje para favorecer un buen rendimiento académico.

En paralelo, también nos interesa validar la escala mediante técnicas multivariantes y análisis factorial confirmatorio con el fin de demostrar que la estructura factorial del instrumento es parsimoniosa y posee un buen ajuste.

\subsection{Hipótesis}

La hipótesis se define en función de la generalización empírica deductiva- inductiva que presenta este estudio y de los objetivos indicados.

En tal sentido, la hipótesis consiste en averiguar si es posible evaluar y definir, al menos, cinco variables del aprendizaje del alumno universitario; por tanto, "conocer si las respuestas de los estudiantes universitarios al cuestionario aplicado definen, al menos, cinco variables de estudio":

$H_{0}$ : No es posible definir cinco variables entre los estudiantes universitarios por las dificultades para establecer estrategias de aprendizaje comunes.

$H_{1}$ : Es posible definir cinco variables entre los estudiantes universitarios para establecer estrategias de aprendizaje comunes.

\subsection{Diseño}

Se emplea un diseño de validación de pruebas (Arribas, 2004; Crocker y Algina, 1986; Escobar y Cuervo, 2008; Prieto y Delgado, 2010; Tovar, 2007), donde se elabora y valida la Escala de Valoración del Aprendizaje del Alumnado Universitario (EVAAU). 


\subsection{Participantes}

Por una parte, se conformó un equipo de siete expertos para validar el cuestionario diseñado (EVAAU) según requisitos de la investigación educativa para la formación de grupos (Escobar y Cuervo, 2008: 29; Cabero y Llorente, 2013), con lo que el rigor es alto de la prueba (Arribas, 2004: 27; Cabero y Llorente, 2013: 14).

Para seleccionar dicho grupo se atendió a diversos criterios, siendo el de homogeneidad el más relevante (Brill, Bishop y Walker, 2006; García y Fernández, 2008): experiencia profesional y académica, profesores universitarios y vinculación con el tema o área de conocimiento a estudiar.

Los expertos revisaron y valoraron los ítems que constituían el cuestionario considerando el nivel de comprensión (claridad) y el grado de pertinencia (representatividad e importancia) de las preguntas (Thomas y Nelson, 2007; Wieserma, 2001), aportando comentarios y sugerencias de mejora cuando evaluaban con puntuaciones menores o iguales que 3. Asimismo, hicieron una valoración global del cuestionario. El resultado final fue un cuestionario formado por 43 ítems en el que los mismos se valoran en una escala tipo Likert, de cinco opciones de respuesta, siendo $1=$ "Muy en desacuerdo" y $5=$ "Muy de acuerdo" (Anexo 1).

Por otra parte, y una vez validado el instrumento de medida a través de juicio de expertos, éste se aplicó, mediante prueba piloto, a una muestra de estudiantes universitarios, de tipo intencional, constituida por 166 alumnos pertenecientes a primer curso del Grado de Primaria de la Facultad de Educación de la Universidad de Las Palmas de Gran Canaria, de ambos sexos, 42 hombres y 124 mujeres, de edades comprendidas entre los 17 y los 46 años para comprobar, a través de indicadores estadísticos, la consideración del cuestionario para su validación psicométrica, tal y como se ha indicado con anterioridad, según índices de ajuste de bondad, coeficientes de fiabilidad y el AFC resultante. La citada muestra goza de un nivel de confianza del 95\%, con una heterogeneidad del 50\% y un margen de error del $7 \%$ para el cálculo muestral de poblaciones finitas, siguiendo los criterios del muestreo probabilístico aleatorio simple, donde la elección de la misma se realiza mediante el azar según el número de componentes previamente definido (Cerón, 2006: 143).

\subsection{Material o Instrumentos}

La propuesta inicial de diseño de la Escala de Valoración del Aprendizaje del Alumnado Universitario (EVAAU) se estructura en una serie de dimensiones en las que se define la orientación del estudiante hacia el aprendizaje según concepción teórica previa.

El contenido de las dimensiones anteriores y las variables que las conforman se trasladan al instrumento EVAAU que se administró a la muestra de estudiantes universitarios objeto de estudio tras la validación por jueces para realizar los diferentes análisis estadísticos y verificar el AFC. Los ítems, algunos reformulados y otros redactados para la ocasión, siguieron la clasificación de las dimensiones predeterminadas (Cuadro 1); como resultado obtuvimos un banco de 43 preguntas que son la base para la elaboración de la versión experimental del cuestionario. 
Cuadro 1. Relación de dimensiones: evaluación del estudiante que orienta el aprendizaje al resultado y al proceso

\begin{tabular}{ll}
\hline Dimensiones & \multicolumn{1}{c}{ Asignación } \\
\hline Estrategia metodológica & $\begin{array}{l}\text { Tareas, operaciones y estrategias que utiliza el estudiante } \\
\text { para preparar la materia de estudio. }\end{array}$ \\
\hline Progreso de los resultados & $\begin{array}{l}\text { Refuerzos del usuario para superar la materia en función } \\
\text { de las calificaciones y la certificación final. }\end{array}$ \\
\hline $\begin{array}{l}\text { Entornos educativos y de } \\
\text { aprendizaje }\end{array}$ & Clima y factores ambientales previos para el aprendizaje. \\
\hline Organización del aprendizaje & $\begin{array}{l}\text { Organización temporal del aprendizaje con antelación } \\
\text { previa. }\end{array}$ \\
\hline Atribuciones en los resultados & $\begin{array}{l}\text { Causas atribuidas por el alumnado hacia la calificación y } \\
\text { resultado final de su proceso de aprendizaje. }\end{array}$ \\
\hline
\end{tabular}

En el proceso de análisis de los ítems se consideraron una serie de indicadores estadísticos, tales como el índice de discriminación y los estadísticos descriptivos de cada una de las preguntas. El proceso de reducción se ha basado en el análisis de estos indicadores mediante los programas estadísticos SPSS 21.0 y AMOS.

\subsection{Procedimiento}

Para diseñar el cuestionario se estableció una serie de requisitos que consideramos indispensables (Alaminos y Castejón, 2006; Anguita et al., 2003; Bisquerra, 2004; Briones, 1998; Martínez Arias, 1995). Por una parte, que el contenido de los ítems fuera relevante y que estuviera acorde con lo que se intentaba medir y evaluar; asimismo, que los enunciados fueran los más claros y precisos, desechando cualquier expresión ambigua o que el lenguaje empleado condujera a la duda; que tuviera un diseño ad hoc; que primase la sencillez en su aplicación; que fuera atractivo en su diseño para facilitar la cumplimentación.

Asimismo, el cuestionario se ha elaborado reuniendo las condiciones de un instrumento de evaluación en las ciencias sociales (Fernández-Ballesteros, 1995; Cronbach, 1990; Muñiz, 1996; Pelechano, 1976, 1988), y el contenido se ha determinado mediante revisión bibliográfica, literatura científica afín al tema y la revisión de expertos (Crocker y Algina, 1986; Del Rincón et al., 1995; McMillan y Schumacher, 2005).

\section{Resultados}

Los datos obtenidos son sometidos, de forma previa, a un Análisis Factorial Exploratorio (AFE) para poder definir el AFC, prestando especial atención a los resultados referidos a los pesos factoriales, fiabilidad e índices de bondad de ajuste y de error. Por tanto, La estructura factorial exploratoria se realizó, por un lado, en base al juicio de los expertos atendiendo a la validez de contenido; y, por otro, una vez clasificados los ítems en sus correspondientes dimensiones procedimos al análisis de fiabilidad y al AFE para atender a la validez de constructo. En este sentido, con el AFE, lo que se 
pretende, es determinar los componentes principales que surgen de rotar las variables investigadas. Así pues, la realización del análisis de componentes principales trata de identificar el número y composición de componentes necesarios para resumir las puntuaciones observadas en las principales variables (Lloret-Segura, Ferreres-Traver, Hernández-Baeza y Tomás-Marco, 2014).

En primer lugar, el análisis de fiabilidad revela que el cuestionario EVAAU presenta un Alpha de Cronbach de 0,805, con lo que la fiabilidad total del instrumento de medida se considera de bastante alta.

En segundo lugar, previo a realizar el AFE, destacamos varias condiciones e indicadores que han confirmado la posibilidad de efectuar dicho análisis:

- Las variables de estudio son cuantitativas, continuas y de intervalo.

- Las variables de estudio se distribuyen normalmente.

- La prueba de esfericidad de Bartlett presenta una significación es 0,000, inferior a 0,05 .

- El test KMO (Kaiser, Meyer y Olkin) revela una puntuación de 0,724, señal que la matriz de correlaciones puede ser factorizada.

Las puntuaciones obtenidas en la diagonal principal de la matriz de correlaciones antimagen se encuentran en el Cuadro 2.

Cuadro 2. KMO y prueba de Bartlett

\begin{tabular}{|c|c|c|}
\hline \multicolumn{2}{|c|}{ Medida de adecuación muestral de Kaiser-Meyer-Olkin. } & ,724 \\
\hline \multirow[t]{3}{*}{ Prueba de esfericidad de Bartlett } & Chi-cuadrado aproximado & 1280,976 \\
\hline & gl & 465 \\
\hline & Sig. & ,000 \\
\hline
\end{tabular}

Determinante $=2,89 \mathrm{E}-007$

Los datos obtenidos confirman que las variables o ítems de estudio cumplen todos los requisitos previos e indicadores para poder realizar el AFE.

Ahora bien, el método de extracción de componentes principales es el empleado para realizar el AFE; como condiciones sin ecuánime se establecen los siguientes criterios de extracción:

a) Criterio de raíz latente. Este criterio señala que solo se obtengan factores con autovalores mayores que 1 . Siguiendo esta condición, la varianza total explicada extrae cinco factores con valores mayores a 1 que explican una varianza total del $67,315 \%$ de la matriz de datos inicial, siguiendo:

b) el criterio de porcentaje de varianza, el cual indica que un buen AFE debe extraer, al menos, un $60 \%$ de la varianza.

- por encima de 0,5, indicativo que la matriz puede ser factorizada. 
Los datos obtenidos confirman que las variables o ítems de estudio cumplen todos los requisitos previos e indicadores para poder realizar el AFE.

Empleamos el método de extracción de componentes principales para realizar el AFE; como condiciones sin ecuánime se establecen los siguientes criterios de extracción: a) Criterio de raíz latente. Éste señala que solo se obtengan factores con autovalores mayores que 1 . Siguiendo esta condición, la varianza total explicada extrae cinco factores con valores mayores a 1 que explican una varianza total del 67,315\% de la matriz de datos inicial, siguiendo; b) el criterio de porcentaje de varianza, el cual indica que un buen AFE debe extraer, al menos, un $60 \%$ de la varianza.

Por tanto, el número de factores extraídos son cinco dimensiones o variables latentes, no directamente observables, que explican el 67,315\% del total de la varianza. Como podemos ver en la tabla 1 que muestra la matriz de componentes del AFE, debemos destacar que los valores obtenidos en las distintas variables que forman las soluciones factoriales extraídas están por encima de 0,50, valor suficiente a considerar y que deben estar las comunalidades para que el AFE sea eficaz (bondad de la solución factorial). Una vez extraídos los datos, lo que hacemos es rotarlos para facilitar su interpretación sin alterar la proporción de varianza explicada por los factores.

Tabla 1. AFE. Matriz de componentes rotados(a)

\begin{tabular}{|c|c|c|c|c|c|}
\hline & & Col & onente & & \\
\hline & 1 & 2 & 3 & 4 & 5 \\
\hline V_28 & 798 & & & & \\
\hline V_42 & ,688 & & & & \\
\hline V_41 & ,687 & & & & \\
\hline V_38 & 683 & & & & \\
\hline V_39 & ,682 & & & & \\
\hline V_20 & ,672 & & & & \\
\hline V_29 & ,659 & & & & \\
\hline V_25 & ,652 & & & & \\
\hline$V_{-} 11$ & ,612 & & & & \\
\hline V_15 & 609 & & & & \\
\hline$V_{-} 14$ & ,526 & & & & \\
\hline$V_{-} 12$ & & 644 & & & \\
\hline V_33 & & 620 & & & \\
\hline V_32 & & 615 & & & \\
\hline V_21 & & 605 & & & \\
\hline V_31 & & 601 & & & \\
\hline V_37 & & ,580 & & & \\
\hline V_26 & & & 783 & & \\
\hline V_27 & & & 705 & & \\
\hline V_36 & & & 629 & & \\
\hline
\end{tabular}




\begin{tabular}{|c|c|c|c|c|c|}
\hline & \multicolumn{5}{|c|}{ Componente } \\
\hline & 1 & 2 & 3 & 4 & 5 \\
\hline V_24 & & & & & \\
\hline V_23 & & & & & \\
\hline V_19 & & & & ,719 & \\
\hline V_17 & & & & ,664 & \\
\hline V_18 & & & & ,628 & \\
\hline V_4 & & & & &, 664 \\
\hline V_9 & & & & & ,632 \\
\hline V_2 & & & & &, 594 \\
\hline V_10 & & & & &, 562 \\
\hline V_35 & & & & &, 548 \\
\hline
\end{tabular}

Método de rotación: Normalización Varimax con Kaiser.

a La rotación ha convergido en 5 iteraciones.

Se extraen 5 factores con cargas factoriales altas, por encima de 0,50. Por tanto, la solución factorial es bastante parsimoniosa, lo que se traduce en que es fácilmente interpretable porque cada variable satura en un factor y cada factor presenta saturaciones altas. Por tanto, esta estructura factorial analizada mediante un AFE, presenta un modelo de cinco factores: estrategia metodológica, progreso de los resultados, entornos educativos y de aprendizaje, organización de los tiempos, atribuciones en los resultados. Esta estructura es, a priori, lo que significa que los resultados del modelo son confirmatorios. En la tabla 2 se pueden observar las principales características descriptivas de cada uno de los elementos de la prueba en los factores establecidos.

Cabe destacar también que la consistencia interna de los factores mostró, en unos, coeficientes de fiabilidad aceptables y, en otros, bastante buenos. El índice utilizado para su comprobación es el Alfa de Cronbach que atiende para su interpretación el baremo clásico establecido por Nunnally (1978), el cual determina la varianza correspondiente. El índice oscila entre los valores 0 y 1 y se considera el instrumento fiable a partir de puntuaciones superiores al umbral de 0,60-0,65. Los valores obtenidos oscilan entre 0,601 y 0,793 tal y como se ilustra en la tabla 3.

Tabla 2. Estadísticos descriptivos de los componentes principales: media, desviación típica, varianza, asimetría y curtosis

\begin{tabular}{lcccccc}
\hline & & $\begin{array}{c}\text { Media } \\
\text { Estadístico }\end{array}$ & $\begin{array}{c}\text { D. T. } \\
\text { Estadístico }\end{array}$ & $\begin{array}{c}\text { Varianza } \\
\text { Estadístico }\end{array}$ & $\begin{array}{c}\text { Asimetría } \\
\text { Estadístico }\end{array}$ & $\begin{array}{c}\text { Curtosis } \\
\text { Estadístico }\end{array}$ \\
\hline \multirow{5}{*}{ Estrategia } & (1) ÍTEM 11 & 3,73 &, 868 &, 754 &,- 469 &, 196 \\
Metodológica & (2) ÍTEM 14 & 3,67 &, 882 &, 778 &,- 330 &,- 291 \\
& (3) ÍTEM 15 & 3,78 &, 926 &, 858 &,- 300 &, 087 \\
& (4) ITEM 20 & 3,45 &, 957 &, 915 &,- 159 &,- 431 \\
& (5) ÍTEM 25 & 3,68 &, 853 &, 728 &,- 282 &,- 191 \\
\hline
\end{tabular}




\begin{tabular}{|c|c|c|c|c|c|c|}
\hline & & $\begin{array}{c}\text { Media } \\
\text { Estadístico }\end{array}$ & $\begin{array}{c}\text { D. T. } \\
\text { Estadístico }\end{array}$ & $\begin{array}{c}\text { Varianza } \\
\text { Estadístico }\end{array}$ & $\begin{array}{l}\text { Asimetría } \\
\text { Estadístico }\end{array}$ & $\begin{array}{c}\text { Curtosis } \\
\text { Estadístico }\end{array}$ \\
\hline \multirow{6}{*}{$\begin{array}{l}\text { Estrategia } \\
\text { Metodológica }\end{array}$} & (6) ÍTEM 28 & 3,81 & ,847 & ,718 &,- 226 &,- 605 \\
\hline & (7) ÍTEM 29 & 3,91 & ,952 & ,907 &,- 542 &,- 426 \\
\hline & (8) ÍTEM 38 & 3,63 & 1,029 & 1,060 &,- 211 &,- 814 \\
\hline & (9) ÍTEM 39 & 3,64 &, 904 & ,816 &,- 233 &,- 686 \\
\hline & (10) ÍTEM 41 & 3,43 & 1,030 & 1,061 &,- 082 &,- 492 \\
\hline & (11) ÍTEM 42 & 3,59 & ,865 & ,749 &,- 937 & 1,395 \\
\hline \multirow{6}{*}{$\begin{array}{l}\text { Progreso de } \\
\text { los resultados }\end{array}$} & (12) ÍTEM 12 & 2,81 & 1,036 & 1,074 & ,085 &,- 433 \\
\hline & (13) ÍTEM 21 & 3,57 & 1,047 & 1,096 &,- 370 &,- 343 \\
\hline & (14) ÍTEM 31 & 2,81 &, 978 &, 957 & ,082 &,- 128 \\
\hline & (15) ÍTEM 32 & 2,74 & 1,003 & 1,005 & , 175 &,- 547 \\
\hline & (16) ÍTEM 33 & 3,36 & 1,012 & 1,024 &,- 409 &, 056 \\
\hline & (17) ÍTEM 37 & 2,88 & 1,023 & 1,046 & ,142 &,- 335 \\
\hline \multirow{5}{*}{$\begin{array}{l}\text { Entornos } \\
\text { educativos } \\
\text { y de } \\
\text { aprendizaje }\end{array}$} & (18) ÍTEM 23 & 4,01 &, 658 &, 533 &,- 302 &, 179 \\
\hline & (19) ÍTEM 24 & 3,96 & ,913 & ,834 &,- 694 & ,449 \\
\hline & (20) ÍTEM 26 & 4,35 & ,730 &, 483 &,- 706 &,- 225 \\
\hline & (21) ÍTEM 27 & 4,21 & ,753 &, 567 &,- 714 &,- 295 \\
\hline & (22) ITEM 36 & 4,49 & ,695 & ,433 & $-1,052$ &, 527 \\
\hline \multirow{3}{*}{$\begin{array}{l}\text { Organización } \\
\text { de los tiempos }\end{array}$} & (23) ITEM 17 & 4,31 & ,848 & 719 & $-1,256$ & 1,390 \\
\hline & (24) ÍTEM 18 & 4,18 & 848 & ,719 & $-1,020$ & 1,250 \\
\hline & (25) ÍTEM 19 & 4,59 & 707 &, 500 & $-2,045$ & 5,062 \\
\hline \multirow{6}{*}{$\begin{array}{l}\text { Atribuciones } \\
\text { en los } \\
\text { resultados }\end{array}$} & (26) ÍTEM 2 & 3,69 & ,830 & ,689 &,- 426 & ,336 \\
\hline & (27) ÍTEM 4 & 2,20 &, 951 & ,905 &, 578 &, 054 \\
\hline & (28) ÍTEM 5 & 3,42 & 874 & ,763 &,- 024 &,- 436 \\
\hline & (29) ÍTEM 9 & 3,07 & 1,227 & 1,505 &, 032 &,- 924 \\
\hline & (30) ÍTEM 10 & 3,39 &, 900 & ,809 &,- 386 & , 185 \\
\hline & (31) ÍTEM 35 & 3,80 & ,798 & ,637 &,- 353 & ,279 \\
\hline
\end{tabular}

Tabla 3. Consistencia interna de los factores o componentes establecidos

\begin{tabular}{lc}
\hline Escalas & Alfa de Cronbach \\
\hline Estrategia metodológica & 0,793 \\
Progreso de los resultados & 0,688 \\
Entornos educativos y de aprendizaje & 0,601 \\
Organización de los tiempos & 0,755 \\
Atribuciones en los resultados & 0,648 \\
\hline
\end{tabular}

Ahora bien, de forma gráfica, los pesos o saturaciones factoriales para cada uno de los ítems que conforman cada uno de los componentes aparecen ilustrados en la figura 1 ; y la tabla 4 permite observar los índices o contrastes de la bondad de ajuste del modelo. 
Figura 1. Saturaciones factoriales correspondientes a los ítems y a las escalas. Máxima verosimilitud. Método de extracción: análisis de componentes principales. 5 componentes extraídos. Método de rotación: Normalización Varimax con Kaiser

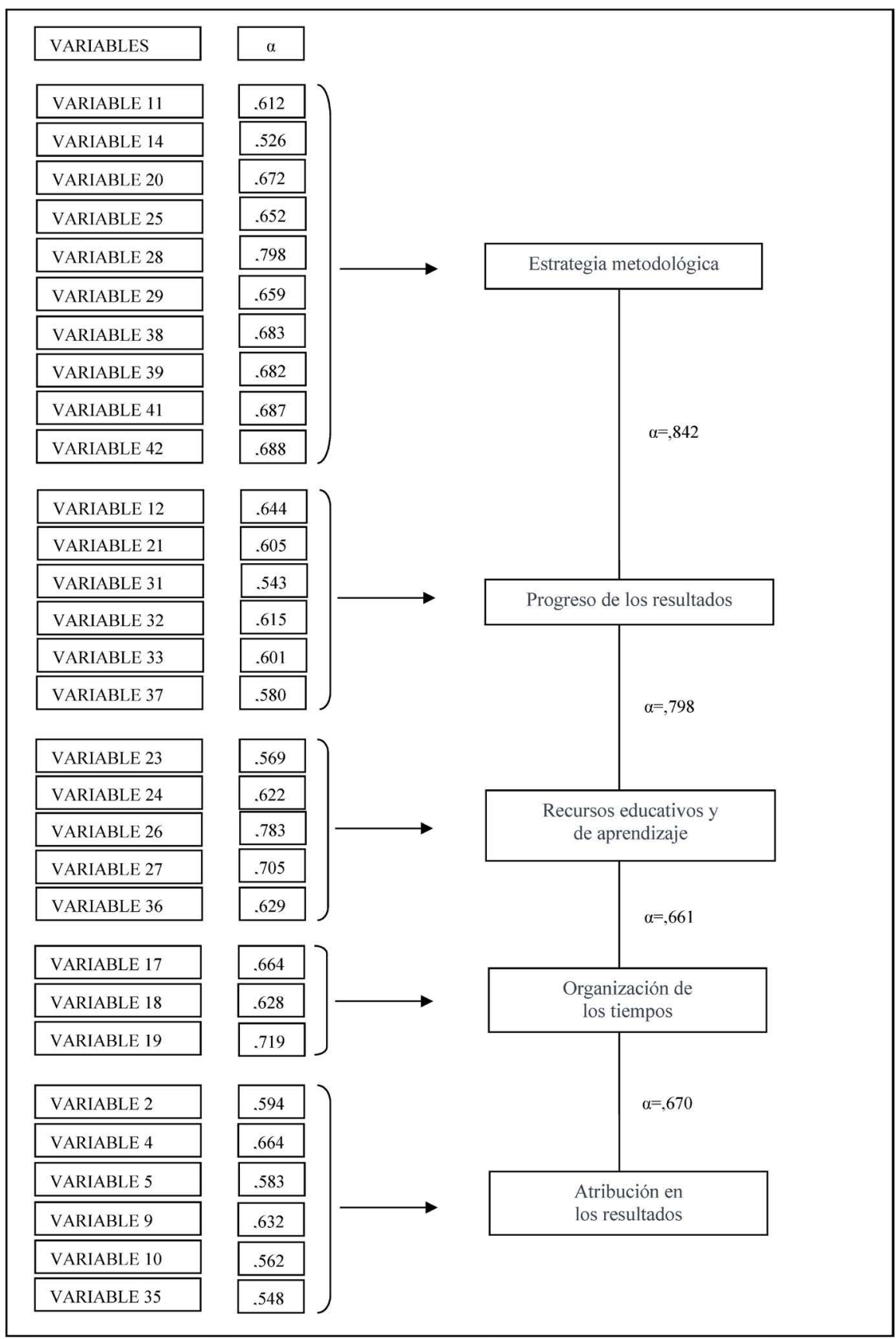


Con los datos expuestos hasta este momento, el análisis factorial confirmatorio (AFC) tratará de valorar el ajuste de los mismos a la estructura que ha sido extraída; por tanto, los citados datos son sometidos a un AFC, prestando especial atención a los resultados referidos a los pesos factoriales, fiabilidad e índices de ajuste (GFI, AGFI) y de error (RMSR y RMSEA) para cada una de las escalas que componen el cuestionario. Así, los resultados en la primera escala, en cuanto a pesos factoriales se encuentran entre 0,52 y 0,79 . De la segunda escala o factor, las saturaciones factoriales se sitúan entre 0,54 y 0,64. Referido al tercer factor los pesos factoriales oscilan entre 0,56 y 0,78 . Los resultados de la cuarta escala se encuentran entre 0,62 y 0,71 . Y el último factor refleja saturaciones que van desde 0,54 a 0,66 . Las saturaciones factoriales superiores a 0,50 en todos los componentes indican correlaciones de las variables y de los factores obtenidos. Los coeficientes de fiabilidad (cargas factoriales) de cada factor fluctúan entre 0,66 a 0,84, lo que confirma que los componentes que integran cada factor resultaron significativos en términos de fiabilidad.

Tabla 4. Contraste de la bondad de ajuste del modelo

\begin{tabular}{llr}
\hline Índices de ajuste y de error & & Valor \\
\hline Chi-cuadrado & Valor & 1188,015 \\
& gl & 494 \\
& Sig. &, 000 \\
GFI & &, 875 \\
AGFI & &, 894 \\
RMSEA & &, 074 \\
RMR & &, 061 \\
Log del determinante de & Matriz no restringida & $-16,656$ \\
& Matriz restringida & $-8,138$ \\
\hline
\end{tabular}

Los datos expuestos en la tabla 4 siguen el modelo de ecuaciones estructurales generado mediante la técnica máxima verosimilitud. Para poder validar y confirmar el modelo se recurre a las principales medidas de ajuste (GFI y AGFI) y de error (RMSEA) y (RMR), según Hair, Anderson, Tatham y Black (1998): a) GFI (Índice de Bondad de Ajuste). Los valores cuanto más cercanos a 1 indican un mejor ajuste; b) AGFI (Índice Ajustado de Bondad). Este es una extensión de GFI. Un nivel aceptable y recomendado es un valor mayor o igual a 0,90. Se le considera índice de ajuste y parsimonia; c) $R M R$ (Residuo Cuadrático Medio). Se utiliza normalmente con matrices de correlaciones. Se pueden considerar como aceptables los valores comprendidos entre 0,05 y 0,08; d) RMSEA (Error de Aproximación Cuadrático Medio). Los valores que pueden considerarse aceptables oscilan entre 0,05 y 0,08. 
Los resultados del AFC reflejados en la tabla 4 señalan que RMSEA=0,074 y $R M R=0,061$, indicativos de un buen ajuste. Asimismo, en lo que respecta a los contrastes de bondad, $\mathrm{GFI}=0,875$ y $\mathrm{AGFI}=0,894$, valores que señalan un buen ajuste. Por su parte, el valor de Chi-cuadrado, que es bajo con una significación asociada de $p=0,00$, permite inferir y aceptar que las posibles restricciones al modelo son correctas.

Por último, los factores generados también se contrastan con la prueba de Escalamiento Multidimensional (MDS) a través de procedimiento Proxscal, para terminar de contrastar la validación empírica de los factores extraídos. El MDS también analiza y justifica la bondad de ajuste del modelo utilizado a través de coeficientes tales como: a) el de Stress de Young y Kruskal, b) el coeficiente de correlación al cuadrado (RSQ), y los coeficientes de Dispersión Explicada (DAF) y el de Congruencia de Tucker (CCT).

La figura 2 y el cuadro 3 indican los datos que se desprenden del MDS.

Figura 2. Análisis MDS de los cinco factores extraídos

\begin{tabular}{|ccc|}
\hline Iteration & S-stress & Improvement \\
1 &, 08547 & \\
2 &, 05333 &, 03214 \\
3 &, 04659 &, 00675 \\
4 &, 04375 &, 00283 \\
5 &, 04355 &, 00020 \\
Iterations stopped because \\
S-stress improvement is less than ,001000 \\
Stress and squared correlation (RSQ) in distances \\
RSQ values are the proportion of variance of the scaled data \\
(disparities) in \\
the partition (row, matrix, or entire data) which is accounted for by their \\
corresponding distances. Stress values are Kruskal's stress formula 1. \\
For matrix \\
Stress = 00401 \\
RSQ = ,99041
\end{tabular}


Cuadro 3. Bondad de ajuste. Medidas de ajuste y stress

\begin{tabular}{|l|r|}
\hline Stress bruto normalizado (SBN) &, 00401 \\
Stress-I &, $05496(\mathrm{c})$ \\
Stress-II &, $12525(\mathrm{c})$ \\
S-Stress &, $00684(\mathrm{~d})$ \\
Coeficiente de correlación al cuadrado (RSQ) &, 99041 \\
Dispersión explicada (D.A.F.) &, 99698 \\
Coeficiente de congruencia de Tucker (CCT) &, 99849 \\
\hline
\end{tabular}

PROXSCAL minimiza el stress bruto normalizado.

c Factor para escalamiento óptimo $=1,003$.

d Factor para escalamiento óptimo $=, 994$.

Los resultados obtenidos revelan coeficientes muy buenos del ajuste del modelo. Las medidas de Stress cercanas a $0(\mathrm{SBN}=0,00401)$ y los indicadores de ajuste que se aproximan a $1(\mathrm{RSQ}=0,99041 ; \mathrm{DAF}=0,9969 ; \mathrm{CCT}=0,99849)$ indican soluciones excelentes.

En definitiva, los diferentes análisis efectuados muestran una calidad métrica satisfactoria de los resultados y, en consecuencia, de los factores extraídos del cuestionario, al ser evaluados mediante procedimientos confirmatorios (ver Anexo 2. Cuestionario EVAAU final). Se ha demostrado un ajuste adecuado del modelo teórico propuesto.

\section{Discusión y Conclusiones}

Los resultados que se han expuesto en este estudio ofrecen una calidad métrica satisfactoria del cuestionario diseñado para evaluar el aprendizaje en alumnos universitarios (EVAAU). Éste ha sido evaluado mediante procedimientos confirmatorios y ha mostrado un ajuste adecuado del modelo teórico previo. Además, muestran una adecuada fiabilidad en términos de adecuación muestral, aplicabilidad y consistencia interna. Así, por ejemplo, en relación a los índices de ajuste y error, todos ellos se encuentran en los valores recomendados para aceptar el modelo como señalan Hu y Bentler (1999).

A la vista de los resultados, y respecto a las estrategias metodológicas, a nivel universitario se debe estimular el aprendizaje de las materias siguiendo estrategias en función del contenido de las asignaturas, y no debe darse por sentado que éstas son utilizadas por el conjunto del alumnado al mismo nivel (Gargallo, Almerich, SuárezRodríguez y García-Félix, 2012; López, González, Aguiar y Artiles, 2017).

En cuanto al progreso de los resultados, y la motivación que genera en el alumnado, es necesario estimular por parte del profesorado una evaluación basada en el aprendizaje (Elizondo, Rodríguez y Rodríguez, 2018), que se apoye en los resultados y en la sensación placentera que ofrece el hecho de conocer y comprender cosas nuevas. Para ello es necesario una interacción profesorado-alumnado dinámica, que 
fomente un entorno educativo y de aprendizaje positiva, dado que las experiencias educativas que estimulan al alumnado están vinculadas a las buenas relaciones con sus docentes estudio (Gallardo y Reyes, 2018). Además, es necesario prestar atención a las atribuciones que realizan los estudiantes sobre sus procesos, así como su autopercepción para regular su aprendizaje (Sevari y Kandy, 2011; Yusuf, 2011).

Por tanto, se puede afirmar que este cuestionario reúne los requisitos metodológicos y psicométricos adecuados y aporta información de gran utilidad para evaluar el afrontamiento del aprendizaje en alumnos universitarios. El cuestionario EVAAU goza de una adecuada validez de contenido y consistencia interna como se ha podido comprobar en los diferentes análisis efectuados. Por ejemplo, la información que revela los estadísticos de fiabilidad y las saturaciones o cargas factoriales de los componentes revelan un más que aceptable coeficiente de confiabilidad. Asimismo, la prueba de la adecuación muestral permite inferir y aceptar que las posibles restricciones al modelo son correctas y la prueba de esfericidad de Bartlett confirma la aplicabilidad del análisis factorial de las variables estudiadas; ambas pruebas señalan la fiabilidad del análisis factorial realizado. Además, los índices de ajuste y de error, todos ellos, se encuentran en los valores recomendados para aceptar el modelo. Estos resultados se ajustan de manera idónea y suficiente a la estructura teórica previa elaborada. Por tanto, se rechaza la hipótesis nula y se acepta la hipótesis alternativa: ha sido posible definir cinco variables entre los estudiantes universitarios para establecer estrategias de aprendizaje comunes.

Sin embargo, esta investigación presenta algunas limitaciones propias de estudios cuyo propósito es determinar la validez del diseño y elaboración de pruebas. Así, por un lado, una recae en la muestra de alumnos universitarios encuestados; la misma no representa a la población universitaria. Por ello, sería conveniente y deseable que, para aumentar la validación del cuestionario, se utilizara una muestra estratificada del conjunto de la población universitaria de la provincia de Las Palmas. Y, por otro, con una muestra más amplia de alumnos se podría determinar, de forma consistente, la influencia de las variables que conforman el cuestionario (afrontamiento del aprendizaje) como elementos predictores (rendimiento académico). Para ello, tendríamos que hallar la validez predictiva de la prueba mediante correlaciones y análisis de regresión múltiple de los datos que se obtuvieran; estos estadísticos procedimentales nos llevarían a configurar una ecuación de regresión, la cual permitiría comprobar si las variables o factores contribuyen de manera significativa a la predicción.

\section{ReFERENCIAS BIBLIOGRÁFICAS}

Alaminos, A. y Castejón, J. L. (2006). Elaboración, análisis e interpretación de encuestas, cuestionarios y escalas de opinión. Universidad de Alicante.

Anguita, J. C., Labrador, J. R., Campos, J. D., Casas Anguita, J., Repullo Labrador, J. y Donado Campos, J. (2003). La encuesta como técnica de investigación. Elaboración de cuestionarios y tratamiento estadístico de los datos (I). Atención primaria, 31(8), 527-538.

Arribas, M. (2004). Diseño y validación de cuestionarios. Matronas profesión, 5(17), 23-29. 
Bisquerra, R. (Coord.). (2004). Metodología de la investigación educativa. Madrid: La Muralla.

Brill, J. M., Bishop, M. J. y Walker, A. E. (2006). The competencies and characteristics required of an effective project manager: A web-based Delphi study. EducationalTechnology Research and development, 54(2), 115-140.

Briones, G. (1998). Métodos y técnicas de investigación para las ciencias sociales. Trillas.

Cabero Almenara, J. y Llorente Cejudo, M. C. (2013). La aplicación del juicio de experto como técnica de evaluación de las tecnologías de la información (TIC). Eduweb. Revista de Tecnología de Información y Comunicación en Educación, 7(2), 11-22. http://tecnologiaedu.us.es/tecnoedu/images/stories/jca107.pdf

Calatayud, F. L. (2017). La Teoría de Metas de Logro como factor de motivación. Un análisis en las clases instrumentales de conservatorio. Revista Electrónica de LEEME, 37, 35-50.

Camarero Suárez, F., Buey, F. M. D. y Herrero Diez, J. (2000). Estilos y estrategias de aprendizaje en estudiantes universitarios. Psicothema, 12(4), 615-622.

Cardozo, A. (2008). Motivación, aprendizaje y rendimiento académico en estudiantes del primer año universitario. Revista de Educación. Laurus, 14, 209-237.

Carrión, J. J. S. (Ed.) (1984). Introducción a las técnicas de análisis multivariable aplicadas a las ciencias sociales (Vol. 9). CIS.

Cerón, M. C. (2006). Metodologías de la investigación social. LOM ediciones.

Coll Salvador, C y Bolea López, E. (1990). Desarrollo psicológico y Educación. Psicología de la Educación, 2, 335-372.

Crocker, L. y Algina, J. (1986). Introduction to classical and modern test theory. New York: Holt, Rinehart and Winston.

Cronbach, L. J. (1990). Essentials of Psychological Testing (5th Ed.). New York: Harper and Row.

Del Rincón, D., Arnal, J., Latorre, A. y Sans, A. (1995). Técnicas de investigación en ciencias sociales. Madrid: Dykinson.

Díaz, F. y Hernández, G. (2002). Estrategias docentes para un aprendizaje significativo. Una interpretación constructivista, 2.

Elizondo, A. E., Rodríguez, J. V. R. y Rodríguez, I. R. (2018). La importancia de la emoción en el aprendizaje: Propuestas para mejorar la motivación de los estudiantes, Cuaderno de Pedagogía Universitaria, 15(29), 3-11.

Escobar-Pérez, J. y Cuervo-Martínez, A. (2008). Validez de contenido y juicio de expertos: una aproximación a su utilización. Avances en medición, 6(1), 27-36.

Fernández-Ballesteros, R. (1995). Cuestiones conceptuales básicas en evaluación de programas. En R. Fernández-Ballesteros (Ed.), Evaluación y programas. Una guía práctica en ámbitos sociales, educativos de salud (pp. 21-47). Madrid: Síntesis.

Freiberg Hoffmann, A., Stover, J. B., de la Iglesia, G. y Fernández Liporace, M. (2013). Correlaciones policóricas y tetracóricas en estudios factoriales exploratorios y confirmatorios. Ciencias Psicológicas, 7(2), 151-164.

Gallardo, G. y Reyes, P. (2018). Relación profesor-alumno en la universidad: arista fundamental para el aprendizaje. Calidad en la Educación, 32, 78-108. 
García, L. y Fernández, S. J. (2008). Procedimiento de aplicación del trabajo creativo en grupo de expertos. Ingeniería Energética, 29(2), 46-50.

García, M. S. (2005). Motive y cree actitudes positivas. En L. M. Villar (Coord.), Programa para la mejora de la docencia universitaria (pp. 79-96). Madrid, PearsonPrentice Hall.

García-Cueto, E. y Miranda, R. (1998). Bondad de ajuste en el análisis factorial confirmatorio. Psicothema, 10(3), 717-724.

García-Valcárcel, A. y Tejedor Tejedor, F. J. (2017). Percepción de los estudiantes sobre el valor de las TIC en sus estrategias de aprendizaje y su relación con el rendimiento. Educación XX1, 20(2), 137-159. https://doi.org/10.5944/educXX1.13447

Gargallo López, B., Garfella Esteban, P. R., Sahuquillo Mateo, P. M., Verde Peleato, I. y Jiménez Rodríguez, M. Á. (2015). Métodos centrados en el aprendizaje, estrategias y enfoques de aprendizaje en estudiantes universitarios: Learning-centered methods, learning strategies and learning approaches in university students. Ministerio de Educación.

Gargallo López, B., Sánchez Peris, F., Ros Ros, C. y Ferreras Remesal, A. (2010). Estilos docentes de los profesores universitarios. La percepción de los alumnos de los buenos profesores. Revista Iberoamericana de Educación, 51(4), 1-16.

Gargallo, B., Almerich, G., Suárez-Rodríguez, J. M. y García-Félix, E. (2012). Estrategias de aprendizaje en estudiantes universitarios excelentes y medios. Su evolución a lo largo del primer año de carrera. RELIEVE. Revista Electrónica de Investigación y Evaluación Educativa, 18(2), 1-22.

Gargallo, B., Suárez-Rodríguez, J. M. y Pérez-Pérez, C. (2009). El cuestionario CEVEAPEU. Un instrumento para la evaluación de las estrategias de aprendizaje de los estudiantes universitarios. RELIEVE. Revista Electrónica de Investigación y Evaluación Educativa, 15(2), 1-31.

Hair, F., Anderson, R. E, Tatham, R. L. y Black, W. C. (1998). Multivariate data analysis with readings (5th edition). New Jersey: Prentice Hall.

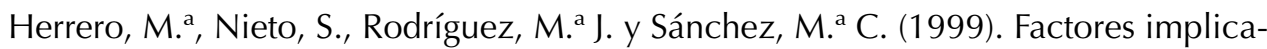
dos en el rendimiento académico de los alumnos de la Universidad de Salamanca. Revista de Investigación Educativa, 17(2), 413-421.

Hu, L. y Bentler, P. M. (1999). Cutoff criteria for fit indices in covariance structure analysis. Structural Equation Modeling, 6, 1-55.

Jöreskog, K. G. (1969). A general approach to confirmatory maximum likelihood factor analysis. Psychometrika, 34, 183-202.

Kaiser, H. J. (1977). Curso básico de estadística: introducción a la técnica descriptiva del análisis estadístico. Herder.

Lloret-Segura, S., Ferreres-Traver, A., Hernández-Baeza, A. y Tomás-Marco, I. (2014). El análisis factorial exploratorio de los ítems: una guía práctica, revisada y actualizada. Anales de psicología, 30(3), 1151-1169.

López, P., González, V., Aguiar, M. V. y Artiles, J. (2017). La gestión de la información en entornos personales de aprendizaje: estudio exploratorio en alumnado de último curso de grado. Revista Complutense de Educación, 28(4), 1303-1320. 
Martínez Arias, R. (1995). Psicometría: Teoría de los tests psicológicos y educativos. Madrid: Síntesis.

McMillan, J. H. y Schumacher, S. (2005). Investigación educativa. Madrid: Pearson.

Monereo, C., Castelló, M., Clariana, M., Palma, M. y Pérez, M. L. (2000). Estrategias de enseñanza y aprendizaje. Graó.

Moral, J. (2006). Análisis factorial confirmatorio. Estadística con SPSS y metodología de la investigación, 445-528.

Morales, P. (2006). Implicaciones para el profesor de una enseñanza centrada en el alumno. Miscelánea Comillas, 64(124), 11-38.

Mosquera, E. D. (2012). Estilos de aprendizaje. Revista Eidos, 5, 5-11.

Muñiz, J. (Coord.). (1996). Psicometría. Madrid: Universitas.

Nunnally, J.C. (1978). Psychometric theory. New York: McGraw-Hill Book.

Padilla-Carmona, M. T. y Gil Flores, J. (2008). La evaluación orientada al aprendizaje en la Educación Superior: condiciones y estrategias para su aplicación en la docencia universitaria. Revista española de pedagogía, 66(241), 467-485.

Pelechano, V. (1976). Psicodiagnóstico. Madrid: Universidad Nacional de Educación a Distancia.

Pelechano, V. (1988). Del psicodiagnóstico clásico al análisis neurológico. Valencia: Alfaplus.

Pérez Gil, J. A., Chacón Moscoso, S. y Moreno Rodríguez, R. (2000). Validez de constructo: el uso de análisis factorial exploratorio-confirmatorio para obtener evidencias de validez. Psicothema, 12(Su2).

Pérez López, C. (2005). Métodos estadísticos avanzados con SPSS. Madrid: Thompson.

Pindyck, R. S. y Rubinfeld, D. L. (2001). Econometria. McGraw-Hill Interamericana.

Prieto, G. y Delgado, A. R. (2010). Fiabilidad y validez. Papeles del psicólogo, 31(1), 67-74.

Rial Boubeta, A., Varela Mallou, J., Abalo Piñeiro, I. y Lévy Mangin, J. P. (2006). El análisis factorial confirmatorio. En J. P. Lévy Mangin (Coord.) y J. Varela Mallou (Dir.), Modelización con estructuras de covarianzas en ciencias sociales: temas esenciales, avanzados y aportaciones especiales (pp. 119-154). A Coruña: Netbiblo.

Rodríguez, J. S. y Remesal, A. F. (2007). Estrategias de aprendizaje y rendimiento académico en estudiantes universitarios. Revista de investigación educativa, 25(2), 421-441.

Sevari, K. y Kandy, M. (2011). Time management skills impact on self-efficacy and academic performance. Journal of American Science, 7(12), 720-726.

Thomas, J. R. y Nelson, J. K. (2007). Métodos de investigación en actividad física. Barcelona: Paidotribo.

Tovar, J. A. (2007). Psicometría: tests psicométricos, confiabilidad y validez. Psicología: Tópicos de actualidad, 85-108.

Uriel, E. y Manzano, J. A. (2002). Análisis multivariante aplicado (Vol. 76). Paraninfo. 
Varela Mallou, J., Abalo Piñeiro, J., Rial Boubeta, A. y Braña Tobio, T. (2006). Análisis Factorial Confirmatorio de segundo nivel. En J. P. Lévy Mangin (Coord.) y J. Varela Mallou (Dir.), Modelización con estructuras de covarianzas en ciencias sociales: temas esenciales, avanzados y aportaciones especiales (pp. 239-258). A Coruña: Netbiblo.

Vivas, N. (2010). Estrategias de aprendizaje. Gondola: Enseñanza Aprendizaje de las Ciencias, 5(1), 27-37.

Wieserma, L. D. (2001). Conceptualization and development of the sources of enjoyment in youth sport questionnaire. Measurement in Physical Education and Exercise Science, 5(3), 153-157.

Ximénez, M. C. y García, A. G. (2005). Comparación de los métodos de estimación de máxima verosimilitud y mínimos cuadrados no ponderados en el análisis factorial confirmatorio mediante simulación Monte Carlo. Psicothema, 17(3). 528-535.

Yusuf, M. (2011). Investigating relationship between self-efficacy, achievement motivation, and self-regulated learning strategies of undergraduate students: a study of integrated motivational models. Procedia Social and Behavioral Sciences, 15, 2614-2617. 


\section{AneXo 1.}

\section{Juicio de expertos. Escala validada}

(1) El alumnado es organizado en líneas generales.

(2) Hay imaginación y creatividad en el alumnado que ingresan en Magisterio.

(3) Estudia habitualmente el alumnado días antes de los exámenes.

(4) Se considera que el rendimiento que logra el alumno en los estudios podría depender de la suerte.

(5) El rendimiento que logra el alumno en los estudios podría depender del profesorado.

(6) Reconoce el estudiante sus puntos fuertes y débiles en el estudio.

(7) Considera el alumno el aprendizaje como lo más importante del estudio.

(8) Las posibles consecuencias negativas que comporta tener malos resultados mueve al alumno a estudiar.

(9) Me han enseñado a estudiar en mi centro de estudio.

(10) Cuando estudio una materia percibo que la carga académica es razonable.

(11) Si obtengo buenos resultados en una materia es porque realizo una síntesis del contenido.

(12) La superación de las materias de la titulación sucede cuando aprendo a través de la memorización.

(13) La superación de las materias de la titulación sucede cuando aprendo a través del razonamiento.

(14) Considero que aprendo de manera independiente, con mis propios medios.

(15) Aprendo mejor cuando la actividad ha sido monitorizada.

(16) Considero que la supervisión de las actividades facilita la motivación.

(17) Pienso que la organización del tiempo optimiza las técnicas de estudio.

(18) Considero que organizar el trabajo diario ayuda a la motivación hacia el aprendizaje.

(19) Pienso que la organización del tiempo de estudio es importante.

(20) El tiempo que dedico al estudio es continuado.

(21) Necesito que refuercen positivamente mis logros.

(22) Cuando reconocen mis logros aumenta mi motivación para estudiar.

(23) Una buena relación con el profesor aumenta la motivación hacia la asignatura.

(24) El interés hacia una asignatura depende del profesor que la imparte.

(25) La motivación hacia una asignatura depende de mi esfuerzo para profundizar en ella.

(26) Un clima de trabajo adecuado favorece la realización de las tareas bien hechas. 
(27) Un clima de trabajo adecuado permite dirigir los pensamientos, sentimientos y acciones hacia la consecución de las metas adecuadas para la formación.

(28) Cuando estudio, elaboro actividades de selección y organización de la información.

(29) Cuando estudio, repaso y realizo la integración de lo nuevo en lo dado.

(30) Siento necesidad de aprender solo por curiosidad por saber.

(31) Mi deseo de aprender se concreta exclusivamente a la consecución de buenas notas.

(32) Estudio por la consecución de recompensas y juicios positivos.

(33) Me interesa la aprobación de mis padres y/o profesores.

(34) No me gusta que realicen valoraciones negativas de mi.

(35) Mi motivación por aprender está en tener más conocimientos.

(36) Me motiva aprender por desarrollar capacidades y competencias.

(37) Considero que el beneficio está en las calificaciones.

(38) Cuando estudio, tengo un esquema de trabajo para procesar la información, adquirir aprendizajes, relacionarlos con los ya adquiridos y aplicar esos conocimientos a la realización de tareas.

(39) Tengo estrategias para aprender cuando me enseñan utilizando distintas situaciones educativas.

(40) Cuando estudio para un examen se me olvida con facilidad lo aprendido.

(41) Preparo los exámenes con tiempo: estudiando con frecuencia las asignaturas, consultando dudas al profesorado, realizando esquemas que me permitan organizar las ideas.

(42) Sé diferenciar las ideas principales de las secundarias y establecer relaciones entre esas ideas.

(43) Me interesa conocer la aplicabilidad y la transferencia del conocimiento que estoy estudiando. 


\section{ANeXo 2.}

\section{Cuestionario para la evaluación del aprendizaje en el alumno universitario}

(1) Hay imaginación y creatividad en el alumnado que ingresan en Magisterio.

(2) Se considera que el rendimiento que logra el alumno en los estudios podría depender de la suerte.

(3) El rendimiento que logra el alumno en los estudios podría depender del profesorado.

(4) Me han enseñado a estudiar en mi centro de estudio.

(5) Cuando estudio una materia percibo que la carga académica es razonable.

(6) Si obtengo buenos resultados en una materia es porque realizo una síntesis del contenido.

(7) La superación de las materias de la titulación sucede cuando aprendo a través de la memorización.

(8) Considero que aprendo de manera independiente, con mis propios medios.

(9) Aprendo mejor cuando la actividad ha sido monitorizada.

(10) Pienso que la organización del tiempo optimiza las técnicas de estudio.

(11) Considero que organizar el trabajo diario ayuda a la motivación hacia el aprendizaje.

(12) Pienso que la organización del tiempo de estudio es importante.

(13) El tiempo que dedico al estudio es continuado.

(14) Necesito que refuercen positivamente mis logros.

(15) Una buena relación con el profesor aumenta la motivación hacia la asignatura.

(16) El interés hacia una asignatura depende del profesor que la imparte.

(17) La motivación hacia una asignatura depende de mi esfuerzo para profundizar en ella.

(18) Un clima de trabajo adecuado favorece la realización de las tareas bien hechas.

(19) Un clima de trabajo adecuado permite dirigir los pensamientos, sentimientos y acciones hacia la consecución de las metas adecuadas para la formación.

(20) Cuando estudio, elaboro actividades de selección y organización de la información.

(21) Cuando estudio, repaso y realizo la integración de lo nuevo en lo dado.

(22) Mi deseo de aprender se concreta exclusivamente a la consecución de buenas notas.

(23) Estudio por la consecución de recompensas y juicios positivos.

(24) Me interesa la aprobación de mis padres y/o profesores.

(25) No me gusta que realicen valoraciones negativas de mí.

(26) Mi motivación por aprender está en tener más conocimientos.

(27) Me motiva aprender por desarrollar capacidades y competencias.

(28) Considero que el beneficio está en las calificaciones. 
(29) Cuando estudio, tengo un esquema de trabajo para procesar la información, adquirir aprendizajes, relacionarlos con los ya adquiridos y aplicar esos conocimientos a la realización de tareas.

(30) Tengo estrategias para aprender cuando me enseñan utilizando distintas situaciones educativas.

(31) Preparo los exámenes con tiempo: estudiando con frecuencia las asignaturas, consultando dudas al profesorado, realizando esquemas que me permitan organizar las ideas.

(32) Sé diferenciar las ideas principales de las secundarias y establecer relaciones entre esas ideas. 